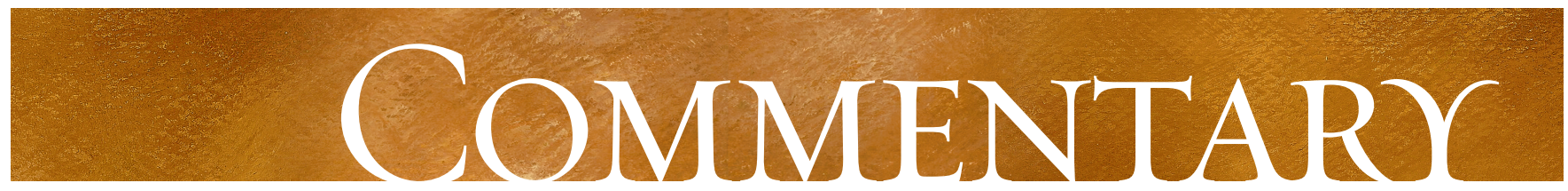

\title{
Do sulfonylurea drugs increase the risk of cardiac events?
}

\author{
David S.H. Bell
}

$\infty \quad$ See related article page 169

S ulfonylureas have been a mainstay for maintaining glucose control in type 2 diabetes. There is ample evidence that maintaining euglycemia decreases the risk of vascular disease in patients with type 2 diabetes; however, there is also evidence that sulfonylurea drugs may actually cause cardiovascular events.

As long ago as 1970, reports have been made of increased cardiovascular mortality with the use of sulfonylureas. The cause of this increase is probably directly related to the mechanism by which sulfonylureas are effective in treating type 2 diabetes. Sulfonylureas in pancreatic $\beta$ cells bind to the subunit of adenosine triphosphate (ATP)-sensitive potassium channels that keeps the channels closed. This causes an influx of calcium ions into the cell that results in an increased release of insulin via exocytosis of insulin-containing granules. Unfortunately, traditional sulfonylurea drugs are not specific for pancreatic $\beta$ cells and also bind to ATP-sensitive potassium channels in cardiomyocytes and vascular smooth-muscle cells.

Channel binding by sulfonylureas in cardiac tissues prevents 3 otherwise beneficial mechanisms: the vascular smooth-muscle cell relaxation that improves coronary blood flow; the limitation of myocardial damage during ischemia; and the protection in cardiomyocytes of energy-generating mitochondria. The phenomenon by which myocardium develops a tolerance to brief periods of ischemia (after which an episode of prolonged ischemia will cause less damage than might otherwise be expected) is collectively known as ischemic preconditioning. ${ }^{1}$ Examples of this preconditioning are the decrease in size of a myocardial infarction when it is preceded by angina, the ability to exercise at the same rate without chest pain after an anginal attack ("warm-up" or "first hole" angina) and the decrease in anginal pain and STsegment depression that accompanies the second balloon inflation during angioplasty. ${ }^{2}$

In this issue of $C M A J$, Simpson and colleagues ${ }^{3}$ present an analysis of administrative data for $4 \mathrm{I} 38$ patients with type 2 diabetes taking glyburide (a more recently developed sulfonylurea) and 1537 , metformin monotherapy. The authors found that an association between higher daily doses and increased risk of death existed with first-generation sulfonylureas (hazard ratio [HR] 2.I, 95\% confidence interval [CI] I.O4.7) and with glyburide (HR I.3, 95\% CI I.2-I.4) but not with metformin (HR 0.8 , 95\% CI 0.7-I.I). This confirms the findings of previous studies that have also reported an increased risk of death with sulfonylurea drugs.
A case-control study ${ }^{4}$ involving patients with type 2 diabetes who received emergency angioplasty revealed that patients taking sulfonylurea drugs had higher rates of death during the first 48 hours (24\%) than those who managed their diabetes with diet alone or with insulin (II\%; $p=0.017)$. When patients have acute coronary syndromes, the detrimental effects of sulfonylureas may be 2 -fold: not only does the sulfonylurea-induced closure of ATP-sensitive potassium channels mitigate the beneficial effects of ischemic preconditioning, ${ }^{4}$ but it may also cause problems with the diagnosis of cardiac ischemia. ST-segment elevation, a hallmark of myocardial infarction revealed by electrocardiography, is caused by changes in the outward potassium current through the ATP-sensitive potassium channels. Closure of these channels can therefore mask ST-segment elevation and deter the administration of potentially life-saving fibrinolytics to the diabetic patient who is taking a traditional sulfonylurea drug. ${ }^{5}$ \section{Sulfonylureas may mask ST-segment elevation during acute coronary syndromes.}

Noninvasive cardiac studies ${ }^{6}$ involving patients without diabetes have shown that the administration of glyburide attenuates the usual improvement in exercise tolerance and myocardial ischemia that occurs when a second stress test is performed an hour after the first. Moreover, in a crossover study ${ }^{7}$ involving patients with diabetes who were known to also have coronary artery disease, the use of glyburide, but not insulin, led to a reduction in left-ventricular ejection fractions during stress testing.

In contrast, a large study ${ }^{8}$ conducted in the United Kingdom on the effects of glycemic control and comparing treatments among people with type 2 diabetes did not find any increase in cardiac events among patients randomly assigned to receive glyburide or chlorpropamide therapy. However, this study excluded patients with established cardiovascular disease. Furthermore, only patients whose diabetes was new- 
ly diagnosed - a group at lower risk of cardiac events than those with a longer history - were included. In addition, the lowering of hemoglobin $\mathrm{A}_{1 \mathrm{c}}$ blood concentrations in the study subjects was suboptimal; prolonged hyperglycemia could therefore have obscured any adverse effect of the sulfonylurea drugs. This is particularly relevant because we now know from the European Prospective Investigation of Cancer (EPICNorfolk) study ${ }^{9}$ that for every $\mathrm{I} \%$ that hemoglobin $\mathrm{A}_{1 \mathrm{c}}$ level exceeds $5 \%$, risk of a cardiac event increases by $26 \%$; and for every $\mathrm{I} \%$ above $7 \%$, it increases by $40 \%$.

Most of the trials that have revealed the adverse effects of ATP-sensitive potassium channel closure in diabetic patients have involved glyburide. ${ }^{10}$ The effects of chlorpropamide or glipizide have not been documented in people, but animal studies suggest that the effects of glipizide are similar to those of glyburide. ${ }^{10}$ Like glyburide, tolbutamide blocks increases in blood flow induced by diazoxide, a vasodilator whose effects are mediated through the opening of ATPsensitive potassium channels. Conversely, the sulfonylurea glimepiride did not exhibit this effect; ${ }^{11}$ furthermore, unlike glyburide, glimepiride does not block the improvements in chest pain and ST-segment depression that usually occur with a second balloon dilation during coronary artery angioplasty. ${ }^{12}$ Glicizide is another example of a sulfonylurea drug that appears to restrict its ATP-sensitive potassium channel activity to the pancreas. Of the meglitinides, it is unlikely that repaglinide, a benzoic acid derivative with a shorter period of attachment to the ATP-sensitive potassium channels, has any advantage over traditional sulfonylureas, since it showed no differences in cardiac events when compared with glyburide during phase 3 studies of repaglinide. On the other hand, nateglinide, a phenylalanine derivative with a very short period of attachment to the K-ATPase receptor, has been shown in animals to have no significant effect on myocardial ATP-sensitive potassium channels.

In conclusion, the findings of Simpson and colleagues ${ }^{3}$ add to the existing evidence that suggests that sulfonylureas increase the risk of cardiovascular events; furthermore, their study adds support to a causal link by demonstrating a doserelated effect on the risk of death. Sulfonylurea drugs should therefore be relegated to third-line agents (after metformin and thiazolidinedione drugs) for managing type 2 diabetes a conclusion also made in recently published guidelines. ${ }^{13}$ If sulfonylurea drugs must be included in a treatment regimen to maintain euglycemia, traditional agents should be avoided; agents such as glimepiride, glicizide and nateglinide, which have less effect on myocardial ATP-sensitive potassium channels,${ }^{10}$ should be prescribed instead.

David Bell is an emeritus professor of medicine at the University of Alabama at Birmingham, Birmingham, Ala.

Competing interests: David Bell is on the speakers' bureaus of GlaxoSmithKline and Aventis Pharmaceutical, and has received fees therefrom.

\section{REFERENCES}

I. Murry CE, Jennings RB, Reimer KA. Preconditioning with ischemic: a delay of lethal cell injury in ischemic myocardium. Circulation I986;74:1124-36.

2. Andreotti F, Pasceri V, Hackett DR, et al. Preinfarction angina as a predictor of more rapid coronary thrombolysis in patients with acute myocardial infarction. $N$ Engl J Med I996;334:7-I2.

3. Simpson SH, Majumdar SR, Tsuyuki RT, et al. Dose-response relation between sulfonylurea drugs and mortality in type 2 diabetes mellitus: a population-based cohort study. CMAJ 2006;174(2):I69-74.

4. Garratt KN, Brady PA, Hassinger NL, et al. Sulfonylurea drugs increase early mortality in patients with diabetes mellitus after direct angioplasty for acute myocardial infarction. J Am Coll Cardiol I999;33:II9-24.

5. Huizar JF, Gonzalez LA, Alderman J, et al. Sulfonylureas attenuate electrocardiographic ST-segment elevation during an acute myocardial infarction in diabetics. J Am Coll Cardiol 2003;42:1017-21.

6. Ovunc K. Effects of glibenclamide, a K(ATP) channel blocker, on warm-up phenomenon in type 2 diabetic patients with chronic stable angina pectoris. Clin Cardiol 2000;23:535-9.

7. Scognamiglio R, Avogar A, Vigili de Kreutzenberg S, et al. Effect of treatment with sulfonylurea drugs or insulin on ischemia-induced myocardial dysfunction in type 2 diabetes. Diabetes 2002;51:808-I2.

8. UK Prospective Diabetes Study (UKPDS) Group. Intensive blood-glucose control with sulfonylureas or insulin compared with conventional treatment and risk of complications in patients with type 2 diabetes (UKPDS 33) [published erratum in Lancet I999;354:602 ]. Lancet I998;352:837-53.

9. Khaw KT, Wareham N, Bingham S, et al. Association of hemoglobin $\mathrm{A}_{\mathrm{c}}$ with cardiovascular disease and mortality in adults: the European Prospective Investigation into Cancer in Norfolk. Ann Intern Med 2004; I4I:413-20.

Io. Bell DSH. Secretagogues and cardiac risk. Endocrinologist 2004;14:33-7.

II. Bijlstra PJ, Lutterman JA, Russel FG, et al. Interaction of sulfonylurea derivatives with vascular ATP-sensitive potassium channels in humans. Diabetologia 1996;39: 1083-90.

I2. Lee TM, Chou TF. Impairment of myocardial protection in type 2 diabetic patients. JClin Endocrinol Metab 2003;88:531-7

I3. Canadian Diabetes Association Clinical Practice Guidelines Expert Committee. Canadian Diabetes Association 2003 clinical practice guidelines for the prevention and management of diabetes in Canada. Can J Diabetes 2003;27(Suppl 2):SI-I40.

Correspondence to: Dr. David S.H. Bell, 5 Io South 20 th St., Rm. 702, Birmingham AL 35294, USA; fax 205 975-9304; dshbell@uab.edu 